\title{
Cataract extraction complicated by capsular glaucoma
}

\author{
ANTHONY J. DARK \\ From the Department of Ophthalmology, Veterans Administration Hospital, Syracuse, New York, USA
}

SUMMARY Synechiae involving the iris pigment epithelium and the peripheral anterior lens capsule occur in capsular glaucoma. During cataract extraction they may bring about traction on the capsule threatening its rupture. The ultrastructural basis of these adhesions is a bonding of pigment cells to capsular surface by pseudoexfoliative material.

Tarkkhanen (1962) noted that the incidence of senile cataract in patients with unilateral pseudoexfoliation (PE) was no greater on the affected than the unaffected side. He concluded that PE disease does not appear to be pathogenically associated with cataract. In contrast, patients with capsular glaucoma have a high incidence of lens opacities. Layden and Shaffer (1974) suggested that some of these cataracts may be iatrogenic as many of these patients have been subjected to filtration procedures and longacting anticholinesterases.

Capsular glaucoma affected about $50 \%$ of Tarkkhanen's (1962) patients with the PE syndrome. The mechanism producing capsular glaucoma is unknown. Since steroid responses are positive in more than $28 \%$ of patients (in contrast to the $90 \%$ seen in chronic simple glaucoma), Pohjola and Horsmanheimo (1971) considered it to be secondary to the PE process. Support for this contention was provided by the transmission electron microscopic (TEM) studies of Ringvold (1971), who found PE material both within and between the trabecular beams of the meshwork and, perhaps more significantly, infiltrating the juxtacanalicular connective tissue as well as within the giant vacuoles of the endothelial cells lining the proximal wall of Schlemm's canal.

It is well known that adequate mydriasis is difficult to obtain in PE disease, a circumstance which is probably due to the extensive infiltration of the iris with PE fibres, as was noted by Ghosh and Speakman (1974). These authors found PE material embedded in the anterior limiting layer, surrounding and even replacing the radial blood vessels as well as being deposited on and between the basement membrane of the iris and pigment epithelium. There is a special tendency for PE fibres in this latter site

Address for reprints: Dr Anthony J. Dark, MD, Department of Ophthalmology, Veterans Administration Hospital, Syracuse, New York 13210, USA to accumulate in the circumferential furrows, where they compress the intervening epithelial ridges, producing a striped appearance macroscopically and a characteristic cogwheel outline in radial microscopic sections as noted by Yanoff and Fine (1975). PE material in this location may cause adherences of the iris to the peripheral lens capsule. Aasved (1973) found fragments of the pigment layer on the anterior lens capsule in such cases, and the 'remnants of posterior synechiae' which Krause and Tarkkhanen (1978) observed in 5 out of 100 extracted cataractous lenses affected with PE disease are presumably further evidence of this type of adhesion.

The nature, extent, and tensility of this bonding of the iris to the periphery anterior lens capsule in capsular glaucoma (and to a less extent in PE disease) has not been the subject of previous comment. The following report describes the histology of these adhesions and indicates that they are a potential cause of capsule rupture when cataract extraction is undertaken.

\section{Material and methods}

Six eyes of 6 consecutive patients with capsular glaucoma were operated on for cataract. Five of the patients had been treated with anticholinesterases for control of glaucoma. Radial iridotomies or sphincterotomies were performed to enlarge the pupil. Alpha-chymotrypsin was used in all cases. The extracted lenses were fixed in cold $2 \%$ glutaraldehyde. Selected specimens of pre-equatorial lens capsule were osmified, dehydrated, and embedded in epoxy resin before sectioning for TEM.

\section{Results}

Preoperative mydriasis in the eye scheduled for cataract extraction was limited to $5 \mathrm{~mm}$ or less in 


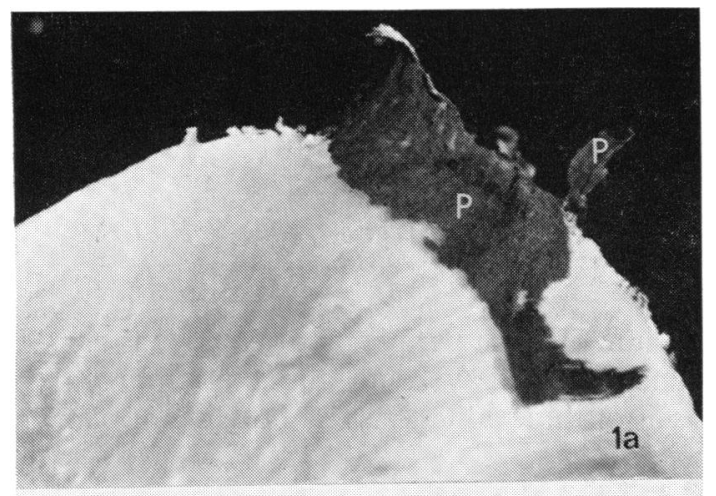

the pigment epithelium of the iris periphery which corresponded in size and position with the pigment sheets adherent to the extracted lenses.

An extensive bonding of the iris pigment epithelium to the pre-equatorial lens capsule by PE fibres was confirmed in TEM (Fig. 3). There was no evidence of collagenous or other fibrous union. Cell membranes of the pigment epithelial cells were often disrupted with PE fibres lying alongside various organelles. In these areas the basement membrane of the iris was also frequently absent. Occasionally bush-like excrescences of PE fibres were capped with ruptured pigment cells (Fig. 4). The lens capsule often showed surface irregularities and multilaminar basement membrane formation (Fig. 3) as well as

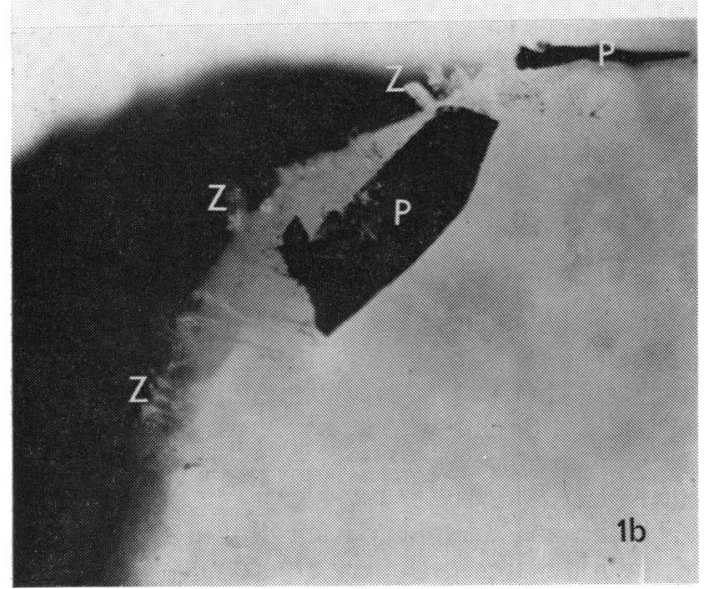

Figs. 1 (a), (b) Sheets of iris pigment epithelium, $P$, are adherent to the pre-equatorial lens capsule. Zonules, $Z$, are encrusted with PE material. Cases 1 and 2

every case. Neither iridodonesis nor phakodonesis was noted in any of these patients. Cataract extraction in all of the patients was protracted and the capsule thrown into traction folds until the lens was finally delivered. The extracted lenses showed 1 to 4 sheets of iris pigment epithelium which were attached to the anterior lens capsule in the preequatorial region (Figs. 1 and 2). In some instances the pigment sheets were rolled over, the posterior iridic surfaces showing the characteristic striping caused by PE fibres accumulated within the circular furrows (Fig. 2). Elsewhere in the pre-equatorial region and to a less extent in the granular zone there was a fine pigment stippling. Pigment granules often adhered to and defined the zonules, which more peripherally were encrusted with PE material. Some zonules were stiffened and stood out from the capsule at an acute angle for a millimetre or so.

Transpupillary transillumination of the irides postoperatively showed sharply defined defects in the pathognomonic deep striated band described by Bertelson et al. (1964).

\section{Discussion}

Impaired mydriasis in patients with senile cataracts may be associated with the PE syndrome. Neverthe-

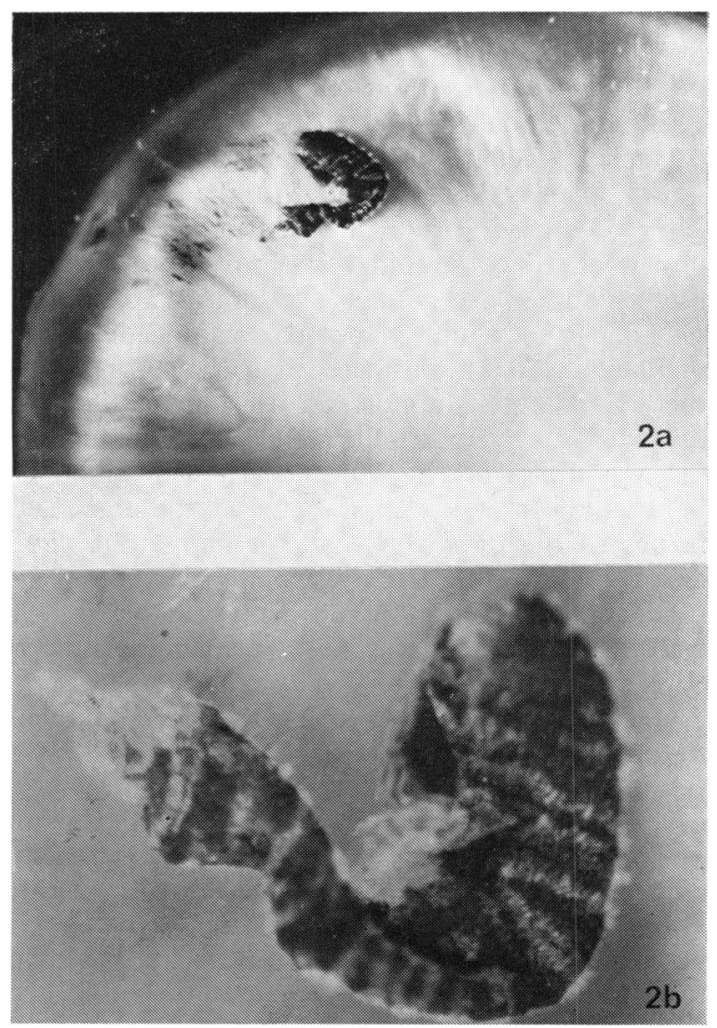

Fig. 2 (a) Iris pigment epithelial sheet adheres to capsule, but it is rolled over to display striped appearance caused by accumulation of $P E$ fibres in circumferential furrows. Case 3. (b): Detail of 2 (a) 
Fig. 3 Pseudoexfoliative fibres, $P E$, bonding iris pigment epithelium, $P$, to zonules, $Z$, and lens capsule, $C$, which shows multilaminar basement membrane formation. The basement membrane of the pigment epithelial cell has almost disappeared and the cell membrane is dehiscent, so that PE fibres lie in direct contact with organelles $(\times 10800)$

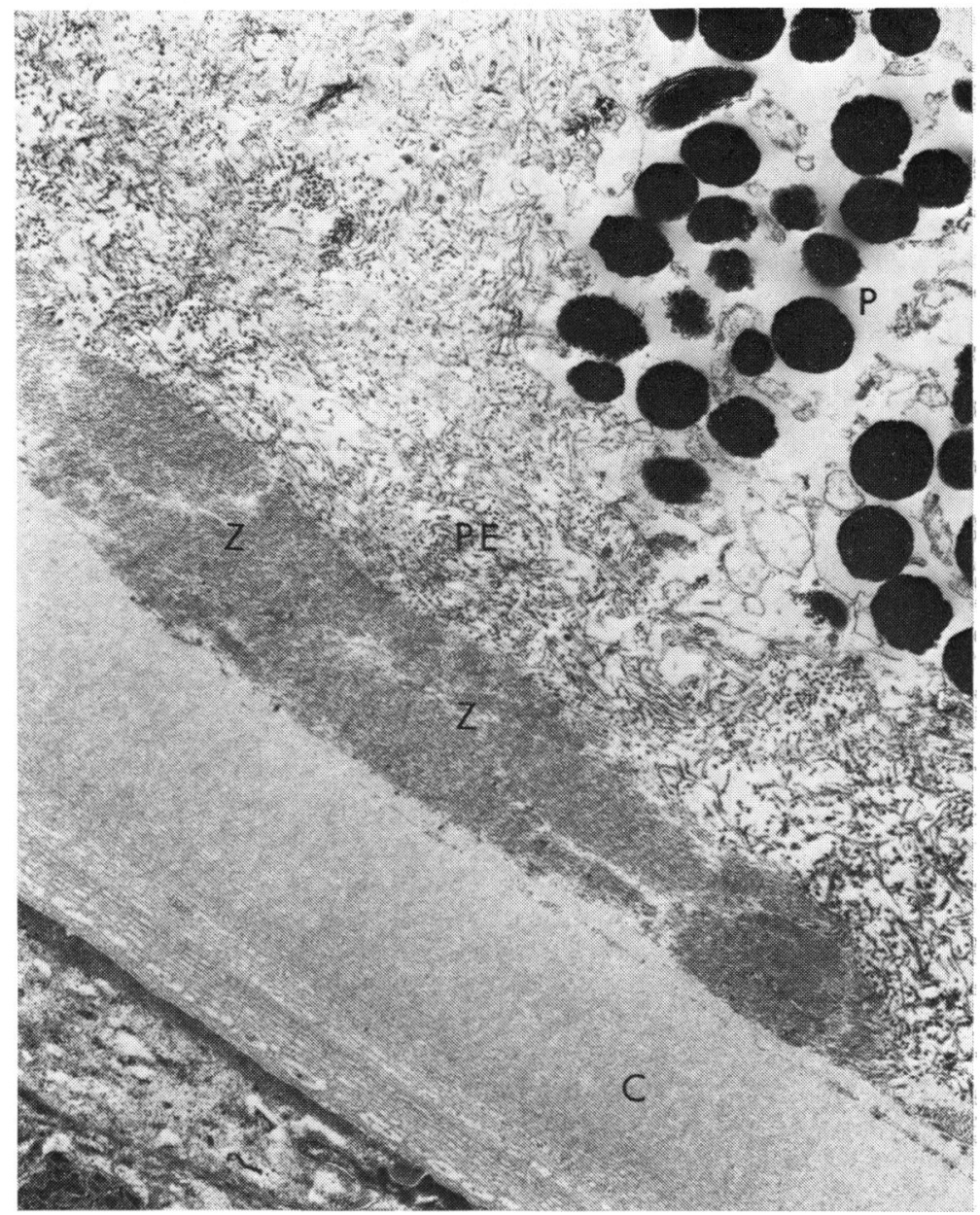

less, it is usually taught that cataract extraction in this disorder is relatively uncomplicated. Certainly the zonules are unduly fragile and may undergo spontaneous rupture, leading to the subluxation of the lens in some patients, as noted by Bartholomew (1970). In contrast, the present study indicates that cataract extraction, in spite of an adequately fashioned pupillary opening, may be difficult in some patients with capsular glaucoma because PE fibres often effect an extensive union of the iris pigment epithelium to the pre-equatorial lens periphery. These adhesions resist lysis with alphachymotrypsin. It is possible, however, that synechiotomies may be performed by sweeping an iris repositor between the lens and iris, a technique which was not used here. Peripheral bonding of the iris to the lens, together with poor dilatation of the pupil after instillation of mydriatic, has also been reported (Zaret et al., 1976) in cataracts which developed after exposure to non-ionising radiant energy. Moreover, difficulty in cataract extraction was also reported in this instance. Finally, it is well recognised that pigment stippling of the anterior lens capsule as well as posterior synechiae and a poor mydriatic response may also be seen in patients who have been treated with long-acting cholinesterases for chronic simple glaucoma. The nature of these adhesions in the 2 latter conditions has not been studied.

Davanger (1977) views PE material as a fibre gel. If so, aqueous could permeate the PE material only by the slow process of diffusion, and hence mass flow would be prevented. PE material deposited between the lens and iris periphery may conceivably impede the flow of aqueous in this way, thus causing a pressure differential between the aqueous compartments resulting in forward movement of the iris with narrowing of the angles. Herbst's (1976) 


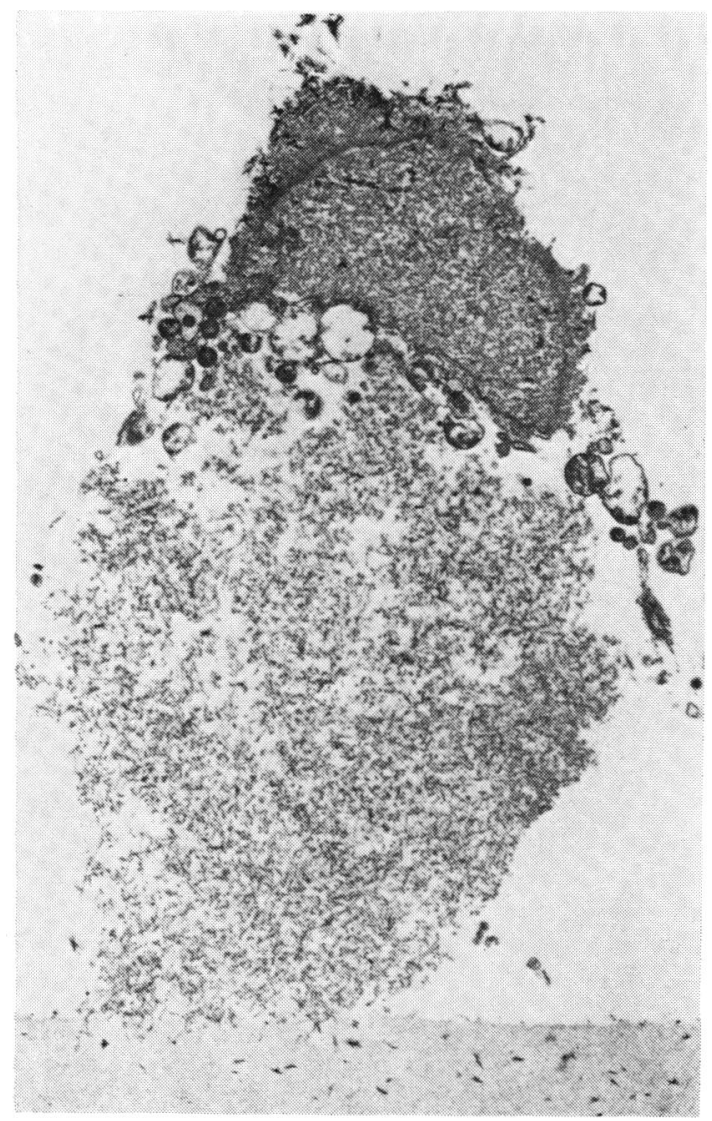

Fig. 4 Bush-like mass of PE fibres capped by disintegrating pigment epithelial cell $(\times 8500)$

patient with unilateral shallow angles and ipsilateral PE disease may have been an instance of such hypothetical mechanics. Layden and Shaffer (1974) found that the incidence of narrow angles (grade 2 or less) in 100 patients with PE syndrome was $25 \%$ in contrast to $5 \%$ of the normal population. These authors point out, however, that many of their patients were on anticholinesterases, which are known to reduce anterior chamber depth. Thus the possible role of these pre-equatorial adhesions in producing angle narrowing in the PE syndrome must for the present remain speculative.

Miss Sharon Edwards was responsible for the technical aspects of microscopy in this report.

This study was supported by a grant from the Medical Research Service of the Veterans Administration.

\section{References}

Aasved, H. (1973). Incidence of defects in the pigmented pupillary ruff in eyes with and without fibrillopathia epitheliocapsularis. Acta Ophthalmologica, 51, 710-715.

Bartholomew, R. S. (1970). Lens displacement associated with pseudocapsular exfoliation. British Journal of Ophthalmology, 54, 744-750.

Bertelson, T. I., Drablos, P. A., and Flood, P. R. (1964). The so-called senile exfoliation (pseudo exfoliation) of the lens capsule, a product of lens epithelium. Acta Ophthalmologica, 42, 1096-1113.

Davanger, M. (1977). On the molecular composition and physiochemical properties of the pseudoexfoliation material. Acta Ophthalmologica, 55, 621-633.

Ghosh, M., and Speakman, J. S. (1974). The iris in senile exfoliation of the lens. Canadian Journal of Ophthalmology, 9, 289-297.

Herbst, R. W. (1976). Angle-closure glaucoma in a patient with pseudoexfoliation of the lens capsule. Annals of Ophthalmology, 8, 853-856.

Krause, U., and Tarkkhanen, A. (1978). Cataract and pseudoexfoliation, a clinico-pathological study. Acta Ophthalmologica, 56, 329-334.

Layden, W. E., and Shaffer, R. N. (1974). Exoliation syndrome. American Journal of Ophthalmology, 78, 835-841.

Pohjola, S., and Horsmanheimo, A. (1971). Topically applied corticosteroids in glaucoma capsulare. Archives of Ophthalmology, 85, 150-153.

Ringvold, A. (1971). Electron microscopy of the trabecular meshwork in eyes with exfoliation syndrome. Virchows Archiv Abteilung A. Pathologische Anatomie, 353, 110-127.

Tarkkhanen, A. (1962). Pseudoexfoliation of the lens capsule. Acta Ophthalmologica, Supplement 71.

Yanoff, M., and Fine, B. S. (1975). Ocular Pathology: A Text and Atlas, p. 365. Harper Row: Maryland.

Zaret, M. M., Snyder, W. Z., and Birenbaum, L. (1976). Cataract after exposure to non-ionising radiant energy. British Journal of Ophthalmology, 60, 632-637. 